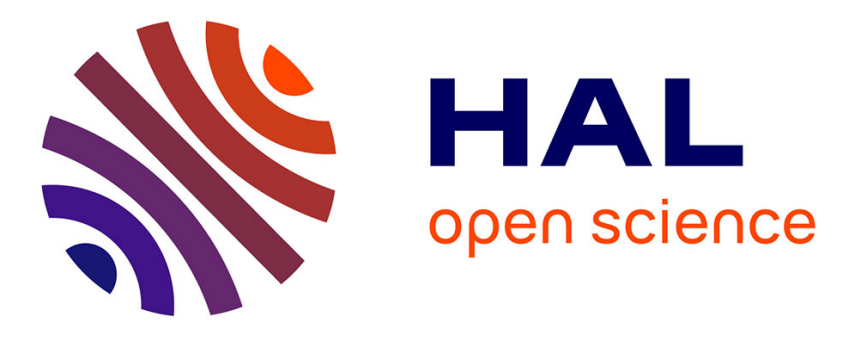

\title{
CoBra: Towards Adaptive Robotized Prostate Brachytherapy under MRI Guidance
}

\author{
Sepaldeep Singh Dhaliwal, Abdelkader Belarouci, Mario Sanz Lopez, Fabien \\ Verbrugghe, Othman Lakhal, Gerald Dherbomez, Taha Chettibi, Rochdi \\ Merzouki
}

\section{To cite this version:}

Sepaldeep Singh Dhaliwal, Abdelkader Belarouci, Mario Sanz Lopez, Fabien Verbrugghe, Othman Lakhal, et al.. CoBra: Towards Adaptive Robotized Prostate Brachytherapy under MRI Guidance. 2021 Sixth International Conference on Advances in Biomedical Engineering (ICABME), Oct 2021, Werdanyeh, Lebanon. pp.81-84, 10.1109/ICABME53305.2021.9604818 . hal-03426965

\section{HAL Id: hal-03426965 https://hal.science/hal-03426965}

Submitted on 16 Nov 2021

HAL is a multi-disciplinary open access archive for the deposit and dissemination of scientific research documents, whether they are published or not. The documents may come from teaching and research institutions in France or abroad, or from public or private research centers.
L'archive ouverte pluridisciplinaire HAL, est destinée au dépôt et à la diffusion de documents scientifiques de niveau recherche, publiés ou non, émanant des établissements d'enseignement et de recherche français ou étrangers, des laboratoires publics ou privés. 


\section{CoBra: Towards Adaptive Robotized Prostate Brachytherapy under MRI Guidance}

\author{
Sepaldeep Singh Dhaliwal (D) \\ CRIStAL UMR CNRS 9189 \\ University of Lille \\ Villeneuve-d'Ascq, France
}

\author{
Abdelkader Belarouci (D) \\ CRIStAL UMR CNRS 9189 \\ University of Lille \\ Villeneuve-d'Ascq, France
}

\author{
Mario Sanz Lopez (1D) \\ CRIStAL UMR CNRS 9189 \\ University of Lille \\ Villeneuve-d'Ascq, France
}

\author{
Fabien Verbrugghe (D) \\ CRIStAL UMR CNRS 9189 \\ École Centrale de Lille \\ Villeneuve-d'Ascq, France
}

\author{
Othman Lakhal \\ CRIStAL UMR CNRS 9189 \\ University of Lille \\ Villeneuve-d'Ascq, France
}

\author{
Gérald Dherbomez (D) \\ CRIStAL UMR CNRS 9189 \\ University of Lille \\ Villeneuve-d'Ascq, France
}

\author{
Taha Chettibi (D) \\ CRIStAL UMR CNRS 9189 \\ University of Lille \\ Villeneuve-d'Ascq, France
}

\author{
Rochdi Merzouki (D) \\ CRIStAL UMR CNRS 9189 \\ University of Lille \\ Villeneuve-d'Ascq, France
}

\begin{abstract}
This paper presents a novel concept for robotized adaptive prostate Brachytherapy (BT) under Magnetic Resonance Imaging (MRI). The Cooperative Brachytherapy (CoBra) concept with compact modular design robot-guide is capable of serving mount of Low Dose Rate (LDR-BT), High Dose Rate (HDR-BT), and Biopsy modules and operate in-bore 3 Tesla MRI. CoBra integrates the multi-components - radiotherapy, imaging, needle, robot-guide as one global system. CoBra MR-robot is a 5 degrees-of-freedom, actuated using non-magnetic piezoultrasonic motors. CoBra Robot intends to place BT seeds to the patient positioned in-bore in lithotomy under MRI-feedback control for the purpose of adaptive brachytherapy. The robot is capable of posing biopsy and BT needle modules for both straight and oblique orientation. It is controlled with an absolute sensor for position sensing. The paper presents recent advances in designing a robotic system for adaptive tumor-targeting in-bore intraoperatively under real-time MRI.
\end{abstract}

Index Terms-Focal therapy, Mechatronics design, MRI Robot, Prostate cancer, Robotized Prostate Brachytherapy

\section{INTRODUCTION}

Prostate cancer is second most commonly diagnosed in men, and it is anticipated that new cases will rise to 1.7 million with 0.5 million possible deaths by 2030 [1]. In conventional practice for Prostate cancer treatment, one of the commonly opted treatment options is low-dose-rate brachytherapy (LDRBT) with trans-rectal ultrasonography imaging modality called TRUS - Brachytherapy (BT). Manual seed placement resulting in LDR-BT accuracy around 3-6 mm [2]. The report by Podder et al. [2] presents various robotized-BT systems based on TRUS and MRI, and nowadays with the advent of technology the MRI is being explored as an alternative option over TRUS for precision and accuracy in oncology due to its superior imaging as compared to TRUS. On the other hand, MRI has its own limitations to work with due to its high magnetic field compatibility issues; and it becomes more challenging for the robotic systems due to conventional actuators and sensors

This work has been co-funded by the European regional development Fund under EU Interreg 2 Seas, Grant CoBra: 2S04-022. which are unsafe under the MRI environment. It is necessary to ensure the compatibility of the components to be used under the MRI environment as per the ASTM standards, described in [3]. A recent survey [4], summarized the proposed robotic solutions for prostate cancer treatment using MRI guidance.

Most of the robots have been explored for the diagnosis to perform biopsy under MRI, only few robots are presented towards LDR-BT under MRI guidance. These robots are further limited to needle positioning with manual needle insertion and dosage delivery by the physician, except [5], [6]. In addition, other limitations factors can be listed:

1) Patient ingress-egress for needle tip verification and insertion.

2) Absence of controlling the robot during the scan within the high field.

3) Action of manual dose delivery.

4) Lack of integration of dosage delivery under real-time (RT) MRI.

5) Absence of adaptation to the prostate motion and edema.

Most of the existing concepts lack intra-operative treatment in-bore real-time delivery, which means they demand patient's out-bore movement for manual needle intervention and moving-in for needle position verification. The reasons can be stated due to patient safety, robot's interference with imaging, for needle tip verification, and complex ergonomic position for a physician to perform an in-bore intervention. To address the above-stated issues and limitations, we explored the gaps and possibilities to get benefited from MRI's RT imaging superiority for intra-operative prostate cancer treatment. Intra-operative imaging can help to achieve closed-loop seed delivery with RT image feedback.

In the framework of the Cooperative Brachytherapy (CoBra) project, the aim is to develop an integrated concept of a robot intended for adaptive BT of the prostate. The CoBra concept intends to adaptively guide the needle holder robot, during the treatment or the diagnosis when the prostate moves or 


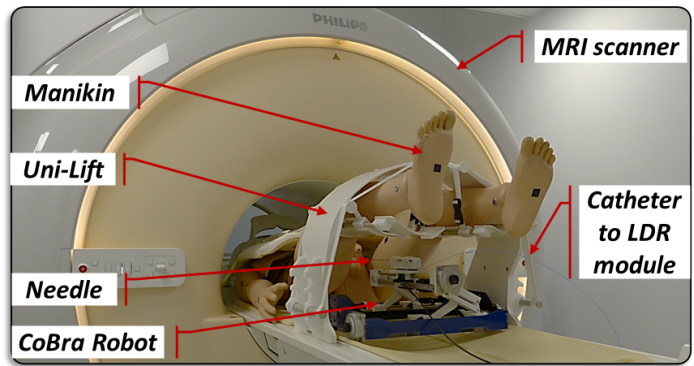

Fig. 1: Description of the environment for an automated brachytherapy of the prostate under MRI.

deforms with the needle contact. Further, the steerable needle adapts to the lesion shift for precise targeting. The intervention takes place in-bore under real-time MRI. The BT module, in terms of performance on the position control of needle insertion for BT, using Heidenhain \& Numeric Jena absolute position sensors (MRI-compatible). The patient is placed in lithotomy position with the aid of MR-safe Uni-Lift device, and needle intervention via trans-perineal skin with minimum access points to reduce the tissue damage and edema, Fig. 1

For intra-operative RT dosimetry accounting the lesion shift occurred during the needle intervention, the targets attained in the pre-operative dosimetry plan are updated intraoperatively with the rapid generation of Pseudo-CT ( $\mathrm{sCT}$ ) data from RTMRI. CoBra uses an AugCGAN - algorithm (augmented cycle Generative Adversarial Network) for sCT data generation [7]. This sCT data is used to update the needle trajectory planning (NPS) software for the dose deposition. The NPS algorithm plan the trajectories based on the minimum number of insertions.

\section{INTEGRATED COBRA CONCEPT}

The robotized brachytherapy of prostate can be stated as multi-component treatment process which integrates different components for overall procedure, such as, $i$ ) Medical Imaging, ii) Dosimetry planning, iii) Needle or applicator module, iv) Radioactive seed/implant, and v) Prostate gland.

Accounting the Prostate gland's anatomy and being a softtissue in nature, makes challenging to target lesions during the intervention as it undergoes deformation and motion. The prostate is surrounded by critical organs such as the urethra, bladder and rectum stated as Organs-at-risk (OARs). Moreover, during the needle intervention, safety must be ensured by avoiding the puncture and seed deposition to the surrounding OARs and healthy tissues. Thus, it demands high precision in terms of targeting the lesion and dose-delivery avoiding excessive dose to healthy tissues or OARs.

The deformation occurred due motion and edema to the prostate during intervention makes it challenging to comply with pre-operative dosimetry plans and deliver seeds to lesion sites. The CoBra-concept proposes an adaptive-BT solution under RT-MRI.

1) Minimizing the number of needle punctures to minimize edema and tissue damage.

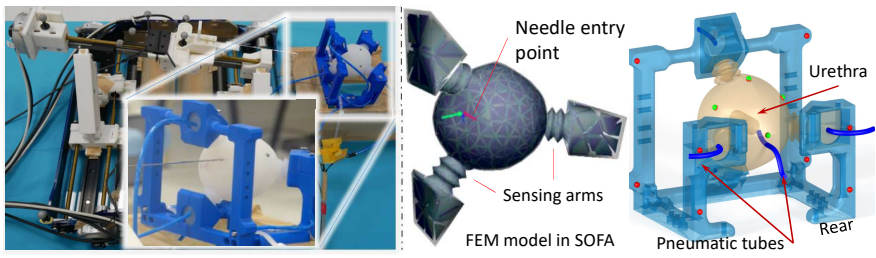

Fig. 2: MRI-safe active prostate phantom - BIP.

2) Intraoperative RT-dosimetry with sCT data generation.

3) Steerable needle for compensating the target shift.

4) Automated seed delivery device coupled to the robot.

5) Reduce the procedural time and cost by maintaining the patient in-bore during the intervention.

6) Improving the BT learning curve and reducing the operator-dependent uncertainties.

7) CoBra ensures lesser image coupling errors, maintaining the same patient position with UNI-Lift for pre-operative imaging and intra-operative imaging.

In order to test and validate the robot-guide, team has developed a bio-inspired prostate phantom (BIP) to mimic the prostate motion and deformation [8], shown in Fig. 2] The BIP is a proof-of-concept as an active prostate phantom, the first of its kind, previously existing commercialized phantoms are static/passive in nature and don't reflect the prostate motion and inflammation occurs during needle intervention. The BIP is coupled with simulation framework SOFA [9], to estimate the deformations based on the interaction forces. BIP is made up of MR-safe materials, e.g., a 3D-printed (PLA) frame and the prostate bulb - Ecoflex silicon 00-30/50.

\section{CoBra RoBot-GUIDE DESIGN}

The design of the CoBra's robotic system for needle module guidance in prostate cancer treatment requires taking into account the following constraints: MRI environment, actuation, sensing, patient positioning, prostate access and workspace. The confined workspace inside MR-bore and specific patient position restrict robot kinematics.

\section{A. Design requirements}

The robot-guide must be capable of operating in-bore within workspace beneath the legs supported by Uni-Lift with lithotomy patient position [10]. The concept should be compatible with 3T wide in-bore scanners, with $70 \mathrm{~cm}$ of diameter, able to include the Uni-Lift leg holder Fig. 1. The prostate access area is through transperineal (TP) in lithotomy preferred over lateral decubitus position. The CoBra concept should make an automatic minimal insertion with 2-3 access points through the perineum, to target multiple lesion sites. This can be possible from oblique insertions over conventional straight needle insertions e.g., [11] concerning the constrained workspace, as shown in Fig. 3. Thus, no template guide is required for needle insertion.

Concerning the key factors for the system, they can be listed as: 1. Active disengagement option for an emergency. 2. Clinician in the loop. 3. Sterilization with replaceable needle option 

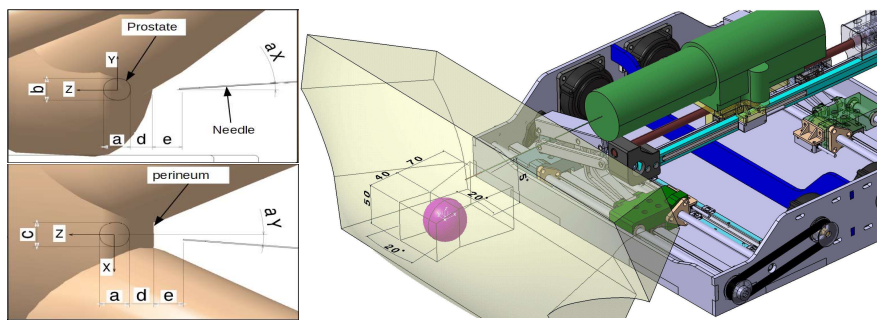

Fig. 3: (A) Workspace constraints, and (B) Robot workspace

TABLE I: Workspace requirements

\begin{tabular}{|c|c|c|c|c|c|c|c|}
\hline $\begin{array}{c}\text { Dimension } \\
\text { range }\end{array}$ & $\begin{array}{c}\mathrm{a} \\
(\mathrm{mm})\end{array}$ & $\begin{array}{c}\mathrm{b} \\
(\mathrm{mm})\end{array}$ & $\begin{array}{c}\mathrm{c} \\
(\mathrm{mm})\end{array}$ & $\begin{array}{c}\mathrm{d} \\
(\mathrm{mm})\end{array}$ & $\begin{array}{c}\mathrm{e} \\
(\mathrm{mm})\end{array}$ & $\begin{array}{c}\mathrm{aX} \\
\left({ }^{\circ}\right)\end{array}$ & $\begin{array}{c}\mathrm{aY} \\
\left({ }^{\circ}\right)\end{array}$ \\
\hline $\min$ & 35 & 30 & 35 & 30 & 30 & -5 & -10 \\
\hline $\max$ & 50 & 40 & 50 & 50 & 50 & 10 & 10 \\
\hline
\end{tabular}

and robot covered using drape. 4. Reduction in edema and trauma/tissue damage with minimal needle insertion through the skin. 5. Accurate needle placement and precise seed delivery under MRI control feedback. 6. Avoidance toxicity to critical biological structures. 7. Adaptation of the dose planning in RT in case of prostate motion.

CoBra's robot-guide intends to operate safely under MRI's strong magnetic filed of 3T, equipped with MR-safe and $M R$ conditional materials with no hazard to patient, staff and without compromising imaging quality of MRI. The robotguide has been tested in-bore real-time under $3 \mathrm{~T}$ and performed normal functioning without getting influenced by the high magnetic field. For that purpose, It has opted for nonferromagnetic actuators and sensors.

\section{B. Workspace constraints}

The prostate gland is beneath the TP skin surrounded by OARs and pubic bone; makes it challenging to target as it is a soft tissue, undergoes deformation and rotation during needle insertion. Moreover, it is challenging for in-bore prostate targeting due to confined space which requires a compact robot and optimal space to access the prostate. The clinically recommended and preferred position is lithotomy Fig. 3 with TP access [10], which helps in accommodating robot and needle module within pyramidal workspace maintaining in-bore patient stability. Workspace constraints illustrated in Fig. 3.A) $: \mathbf{a}, \mathbf{b}$ and $\mathbf{c}$ are the average dimensions of the prostate, $\mathbf{d}$ the distance between the perineum and the prostate, $\mathbf{e}$ the distance between the needle tip and the perineum. $\mathbf{a X}$ and $\mathbf{a Y}$ are the needle angle ranges for oblique insertion along the horizontal and vertical axis, Table I] Fig. 3(B) shows CoBra robot-guide workspace with respect to the prostate. Fig. 4 illustrates the global scenario of in-bore patient and robot placement.

\section{TRajectory Planning BASEd ON Dosimetry}

For the cancer treatment, a dosimetric plan is generated to deliver the dose to lesion sites as focal therapy or whole prostate gland targeting the required dose to the cancer sites. The geometric coordinates are prepared for seed deposition with treatment planning software. For the CoBra robot, the

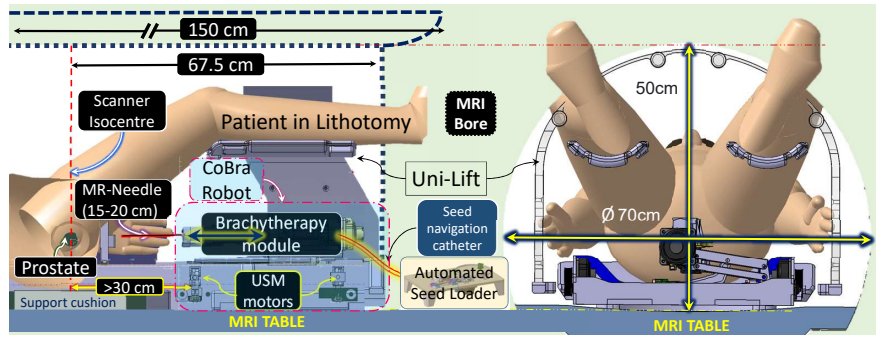

Fig. 4: Placement of patient and robot-setup in-bore 3T MRI.

NPS is developed with the minimal number of needle insertions targeting minimal invasive treatment [12]. The NPS takes the input of the seed-plan to generate the seed deposition coordinates, this helps the software to generate needle trajectories (straight and oblique) with the minimum number of transperineal entry points. NPS can generate entry points (1 to 3 ) as per requirement depending upon the patient prostate size and dose plan, and the physician finalizes the number of suggested puncture points. The software is capable of calculating the minimal tissue damage to reduce the edema effect during the treatment, eventually which will help in reducing the post-surgery seed drift. The NPS works as an adaptive trajectory planning software and accounts for the real-time/true seed positions of delivered seeds, which means it accumulates the relative dose-planning of the drifted or dragged seeds during delivery in soft-tissues. The NPS coupled with the robot's trajectory planning module help in adaptive closed-loop control.

\section{A. Dosimetry under CoBra concept}

For LDR-BT, dosimetric planning consists of defining the required geographic coordinates of the intended radioactive seed implantation position, based on the patient's anatomy and allowed seed positions, to obtain the desired dose distribution [13]. The treatment plan should ensure that the prostate receives a radiation dose high enough to destroy cancer while sparing healthy surrounding tissues and the internal urethral sensitive structure as far as possible. Planning using the MRI images can improve dose planning, improving target coverage, and reduce the dose to OARs [14].

In clinical practice, based on TRUS imaging for LDR dosimetric planning; OARs are contoured from prostate Ultrasound (US) imaging. Based on the anatomical model and pre-defined dose-tolerances an optimization of needle and seed positions is performed. New positions of needles are updated manually (within the software) under live US image-guidance. As small inaccuracies in seed placement corresponding to planned dose can affect the overall dosimetric plan. In some cases, seeds may drift due to needle and soft tissue interaction from the planned delivery sites. Another is the imaging limitation of the US to determine the seeds true position. Considering such issues the CoBra concept based on the MRI accounts deeplearning approach to generate pseudo-CT data-sets, briefed in the following paragraph. 


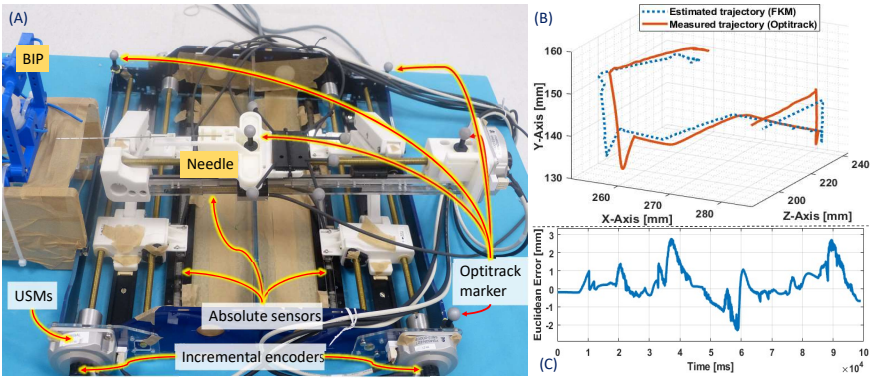

Fig. 5: (A) Robot-guide; (B) 3D Trajectory reconstruction; (C) Euclidean Error.

As in radiotherapy for dosimetric calculations the treatment planning system demand directly the pixel values corresponding to the electron density for attenuation calculation, which is not currently possible with MR-imaging. To formulate workflow based on MR-images only, the MR-images are required to be converted to pseudo-CT ( $\mathrm{sCT}$ ). For the CoBra project, Boni et al. [7] has used a deep-learning approach using MR-images data-sets from different medical centres used for training of neural-network generating sCT from T2-weighted MR-images. A review presented by $\mathrm{Hu}$ et al. [15], states about the advancements and use of deep-learning for prostate brachytherapy with lesion delineation, automatic segmentation and reconstruction of implant needle, and optimization in real-time for treatment planning with dose-calculation. Mao et al. [16] presented a deep-learning-based approach resulting in faster calculations than Monte Carlo resulting in similar accuracy, which can be helpful in intra-operative brachytherapy.

\section{Results And CONClusion}

During the preliminary experiments on the robot, kinematic model has been validated through the Optitrack ${ }^{\mathrm{TM}}$ Prime 13 cameras, marker setup on robot shown in Fig. 5(A), streaming the tracking data to robot-guide directly. The needle tip measurements obtained from the Optitrack ${ }^{\mathrm{TM}}$ were compared with those of the forward kinematic model 5 (B). Euclidean error obtained is presented in Fig. 5(C). The mean Euclidean error for the curve reconstruction is $\approx 2.65 \mathrm{~mm}$ without robot calibration step. For future steps, an online calibration of the robot, to minimize the parameter uncertainties from mechanical design will be finalized, before performing tests of the insertions of the needles through first bio-inspired phantoms and second on animals. Fig. 6 shows CoBra concept's functional compatibility tests under in-bore real-time 3 T MRI. In future work, we will also integrate the automated seedloader along with the straight and oblique needle trajectories to deposit LDR seeds and establish the closed-loop control validating it on the BIP, and followed by the in - vivo tests.

\section{ACKNOWLEDGMENT}

Authors thank partners and observers for assisting with components, TPS by Eckert \& Ziegler BEBIG, Uni-Lift by NORAS, MR-guidewire by MaRVis Tech, flexible catheter by Zeus and absolute encoder by Heidenhain \& Numeric Jena.

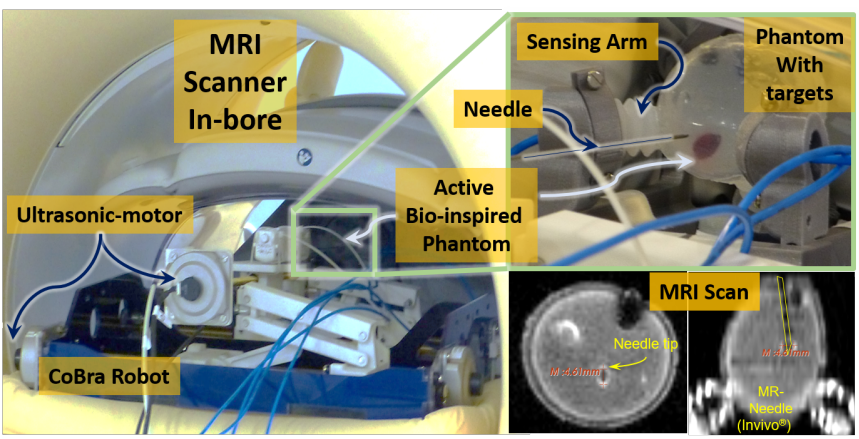

Fig. 6: CoBra robot and BIP in-bore RT testing under 3T MRI.

\section{REFERENCES}

[1] https://www.iarc.who.int/wp-content/uploads/2018/07/pr209_E-1.pdf

[2] T. K. Podder, L. Beaulieu, B. Caldwell, R. A. Cormack, J. B. Crass, A. P. Dicker et al., "AAPM and GEC-ESTRO guidelines for image-guided robotic brachytherapy: Report of Task Group 192," Medical Physics, vol. 41 , no. 10,2014

[3] D. S. Stoianovici, C. Kim, G. Srimathveeravalli, P. Sebrecht, D. Petrisor, J. A. Coleman, S. B. Solomon, and H. Hricak, "MRI-Safe Robot for Endorectal Prostate Biopsy," IEEE/ASME Transactions on Mechatronics, vol. 19, pp. 1289-1299, 2013.

[4] S. S. Dhaliwal, T. Chettibi, S. Wilby, W. Polak, A. L. Palmer, N. Reynaert et al., "Review of Clinical and Technological Consideration for MRI-Guided Robotic Prostate Brachytherapy," IEEE Transactions on Medical Robotics and Bionics, vol. 3, no. 3, pp. 583-605, 2021.

[5] M. Muntener, A. Patriciu, D. Petrisor, M. Schar, D. Ursu, D. Y. Song, and D. Stoianovici, "Transperineal prostate intervention: robot for fully automated MR imaging - system description and proof of principle in a canine model," Radiology, vol. 247, no. 2, pp. 543-549, 2008.

[6] G. Li, H. Su, W. Shang, J. Tokuda, N. Hata, C. M. Tempany, and G. S. Fischer, "A fully actuated robotic assistant for MRI-guided prostate biopsy and brachytherapy," Medical Imaging 2013: ImageGuided Procedures, Robotic Interventions, and Modeling, vol. 8671, p. 867117, 2013.

[7] K. B. B. Boni, J. Klein, L. Vanquin, A. Wagner, T. Lacornerie, D. Pasquier, and N. Reynaert, "MR to CT synthesis with multicenter data in the pelvic era using a conditional generative adversarial network." Physics in Medicine \& Biology, 2020.

[8] S. E. Navarro, S. S. Dhaliwal, M. S. Lopez, S. Wilby, A. L. Palmer, W. Polak et al., "A Bio-Inspired Active Prostate Phantom for Adaptive Interventions," IEEE Transactions on Medical Robotics and Bionics, 2021. [Online]. Available: http://doi.org/10.1109/TMRB.2021.3123385

[9] "SOFA," https://www.sofa-framework.org/ (Accessed on 07/08/2021).

[10] S. S. Dhaliwal, T. Chettibi, A. Belarouci, G. Dherbomez, V. Coelen, and R. Merzouki, "Cooperative Brachytherapy for Prostate Cancer Under MRI Guidance," in 2019 Fifth International Conference on Advances in Biomedical Engineering (ICABME), 2019, pp. 1-4. [Online]. Available: http://doi.org/10.1109/ICABME47164.2019.8940278

[11] R. Merzouki et al., "Curiethérapie," https://www.youtube.com/watch?v= 3f41dV8EIT4\&feature=youtu.be\&t=192. (Accessed 08/21/2020).

[12] S. S. Dhaliwal, S. Wilby, S. Firouzy, K. B. Boni, M. de Vries, S. E. Navarro et al., "CoBra robot for localized cancer treatment and diagnosis under real-time MRI," AUTOMED 2021, June 2021. [Online]. Available: http://doi.org/10.5281/zenodo.4923036

[13] K. Tanderup, A. N. Viswanathan, C. Kirisits, and S. J. Frank, "Magnetic Resonance Image Guided Brachytherapy," Seminars in Radiation Oncology, vol. 24, no. 3, pp. 181,191, 2014-07.

[14] S. P. Register, R. Kudchadker, L. Levy, D. Swanson, T. Pugh, T. Bruno, and S. Frank, "An MRI-based dose-reponse analysis of urinary sphincter dose and urinary morbidity after brachytherapy for prostate cancer in a phase II prospective trial." Brachytherapy, vol. 12 3, pp. 210-6, 2013.

[15] H. Hu, Y. Shao, and S. Hu, "A Review of the Application of Deep Learning in Brachytherapy," Open Access Library Journal, vol. 7, pp. $1-9,2020$. 
[16] X. Mao, J. Pineau, R. Keyes, and S. Enger, "RapidBrachyDL: Rapid Radiation Dose Calculations in Brachytherapy via Deep Learning.' International Journal of Radiation Oncology • Biology • Physics, 2020. 